\title{
Barrier Trees of Degenerate Landscapes ${ }^{1}$
}

\author{
Christoph Flamm ${ }^{a, *}$, Ivo L. Hofacker ${ }^{a}$, Peter F. Stadler $^{a, b}$, \\ Michael T. WolfingeR ${ }^{a}$
}

${ }^{a}$ Institute for Theoretical Chemistry and Molecular Structural Biology, University of Vienna, Währingerstrasse 17, A-1090 Vienna, Austria E-Mail: $\quad$ xtof, ivo, studla,mtw\}@tbi.univie.ac.at URL: http://www.tbi.univie.ac.at/ $\{$ xtof, ivo, studla,mtw $\}$ ${ }^{b}$ The Santa Fe Institute, 1399 Hyde Park Road, Santa Fe, NM 87501, USA

\begin{abstract}
The heights of energy barriers separating two (macro-)states are useful for estimating transition frequencies. In non-degenerate landscapes the decomposition of a landscape into basins surrounding local minima connected by saddle points is straightforward and yields a useful definition of macro-states. In this work we develop a rigorous concept of barrier trees for degenerate landscapes. We present a program that efficiently computes such barrier trees, and apply it to two well known examples of landscapes.
\end{abstract}

Keywords: Fitness landscape, Potential energy surface, energy barrier, saddle points, degenerate states

\footnotetext{
${ }^{1}$ Dedicated to Peter Schuster on the occasion of his 60 th birthday.
} 


\section{Introduction}

The relationship between dynamics and the energy or fitness landscape is indispensable for the understanding of a wide range of phenomena from biopolymer folding, global optimization, and properties of disordered spin systems. A thorough understanding of the geometric details of landscape structure is thus of wide interest. The scenario that emerges e.g. from the replica approach to disordered spin systems is that the phase space is broken into many valleys [22]. These valleys are connected by saddle points.

Methods to elucidate the basin structure of landscapes by means of trees that represent local minima and their connecting saddle points have been developed independently in different contexts, among them $\pm J$ spin models [17, 16], potential energy surfaces (PES) for protein folding [2, 10] and molecular clusters [30, 7], and the kinetics of RNA secondary structure formation [9].

Local minima, saddle points, basins and related concepts are readily defined in nondegenerate landscapes. The definitions are largely consistent between the discrete models such as RNA and the smooth PES in $\mathbb{R}^{n}$. For example, in PES saddle points can be identified as transition states. In the presence of degeneracies, however, the situation becomes more complicated, and additional care has to be taken to find suitable definitions. As a trivial example, consider the flat landscape. Is every point a minimum, or none? If the second alternative is chosen, then the global minimum is not a minimum; clearly one would like to avoid such a strange language. Two non-adjacent minima should, in our intuition, be separated by one or more saddle points. But all points are minima in flat-land, so saddles can be minima as well. This is also counter-intuitive.

The purpose of this contribution is two-fold. In the following two sections we discuss some definitions for concepts related to saddles and basins as well as their mutual relationships. Furthermore we give a formal definition of the barrier tree and the associated basin structure in arbitrary landscapes. In the second part of this manuscript we describe the software package barriers for computing all saddle points in an arbitrary landscape. We illustrate our discussion with a number of examples from two different classes of landscapes.

\section{Degeneracies in Landscapes}

2.1. Notation. We write $\subset$ and $\subseteq$ to distinguish between proper subsets and subsets including the complete set. Throughout this contribution we will consider $G(V, E)$ to be a connected, undirected, simple graph with vertex set $V$ and edge set $E$. For the neighbors (adjacent vertices) of $x \in V$ we write

$$
\partial x=\partial\{x\}=\{y \in V \mid\{x, y\} \in E\}
$$

for the neighbors (adjacent vertices) of $x \in V$. This definitions extend in a natural way to arbitrary vertex sets:

$$
\partial A=\{y \in V \backslash A \mid \exists x \in A:\{x, y\} \in E\}
$$



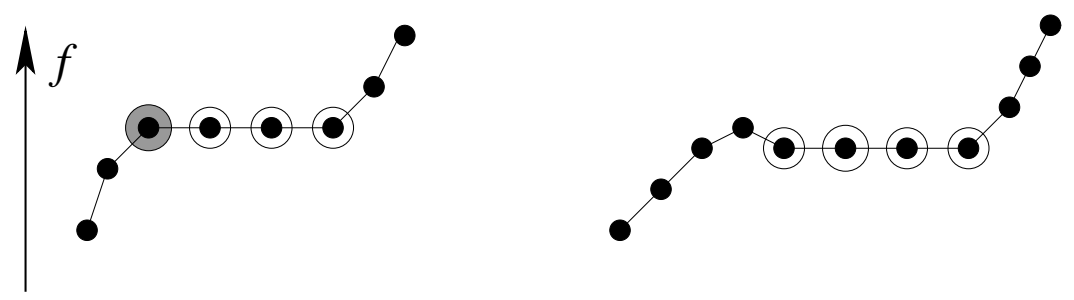

Figure 1. Two types of local minima: shoulder (l.h.s) and valley (r.h.s.). Local minima are marked by white circles. The vertex marked in gray is a saddle point but not a minimum of the landscape.

The set $\partial A$ is the boundary of $A$. Furthermore, we write $\bar{A}=A \cup \partial A$ for the graphtheoretic closure of a vertex set $A \subseteq V$. The neighborhood of $x$ is $N(x)=\{x\} \cup \partial x=$ $\overline{\{x\}}$.

Definition 1. A landscape $(G, f)$ on a graph $G(V, E)$ is a function $f: V \rightarrow \mathbb{R}$.

The graph $G$ is oftentimes referred to as the configuration space in the context of combinatorial landscapes. We write $\min f$ for the value of the global minimum of the fitness function.

2.2. Local Minima. A vertex $x$ is a local minimum of $f(x) \leq f(y)$ for all $y \in \partial x$ (or, equivalently, $y \in N(x)$ ); $x$ is a strict local minimum if $f(x)<f(y)$ for all $y \in \partial x$. We write $\mathcal{M}$ for the set of all local minima. Furthermore, let $\mathcal{M}(x)$ be the vertex set of the connected components of $G[\mathcal{M}]$ that contains $x$. Of course $f$ is constant on $\mathcal{M}(x)$. The set of these components is denoted by $\mathfrak{M}=\{\mathcal{M}(x) \mid x \in \mathcal{M}\}$.

There are two classes of local minima that can be distinguished by the behavior of the function $f$ on $\partial \mathcal{M}(x)$, see Fig. 1 .

(1) $\mathcal{M}(x)$ is a valley if $f(y)>f(x)$ for all $y \in \partial \mathcal{M}(x)$.

$(2) \mathcal{M}(x)$ is a shoulder if there is a $y \in \partial \mathcal{M}(x)$ such that $f(y)=f(x)$.

Definition 2. Consider a landscape $f$ on $G(V, E)$. We say $(G, f)$ is

non-degenerate or invertible if $f(x)=f(y)$ implies $x=y$ for all $x, y \in V$; locally invertible if $x, y \in N(z)$ and $f(x)=f(y)$ implies $x=y$ for all $z \in V$; non-neutral if $f(x)=f(y)$ and $y \in N(x)$ implies $x=y$ for all $y \in V$;

We collect a number of obvious consequences of definition 2 in the following

Lemma 3. (1) non-degenerate $\Longrightarrow$ locally invertible $\Longrightarrow$ non-neutral

(2) If $(G, f)$ is locally invertible then the end-point of a gradient walk is uniquely determined by its initial condition. (3) All local minima are strict in non-neutral landscapes.

Let us briefly consider a few examples of landscapes. Non-degenerate landscapes are for example all generic instances of $p$-spin Hamiltonians

$$
\mathcal{H}(\vec{x})=\sum_{i_{1}<i_{2}<\ldots i_{p}} J_{i_{1} i_{2} \ldots i_{p}} x_{i_{1}} x_{i_{2}} \ldots x_{i_{p}} \quad x_{k}= \pm 1
$$


with odd values of $p$ and $J_{\ldots}$ drawn from a continuous distribution function. Even values of $p$ imply the symmetry $\mathcal{H}(\vec{x})=\mathcal{H}(-\vec{x})$ and generically every value of $\mathcal{H}(\vec{x})$ appears exactly twice. Even $p$-spin models generically have locally invertible landscapes for $n>2$ spins because the maximum Hamming distance of configurations in the neighborhood of a point is 2 while the $d_{H}(\vec{x},-\vec{x})=n$. The landscape of $\mathcal{H}(\vec{x})=\sum_{i=1}^{n} x_{i}$ is non-neutral because the change $x_{i} \rightarrow-x_{i}$ changes $\mathcal{H}(\vec{x})$ by 2 , i.e., neighboring configurations always have different values of $\mathcal{H}$. However, if $x_{i}=x_{j}$ then flipping either $i$ or $j$ leads to the same value of $\mathcal{H}$ for both mutants, thus $H$ is not locally invertible. The fitness landscapes based on RNA folding discussed e.g. in [25] show a large number of neutral neighbors.

2.3. Walks. A walk $\mathbf{p}$ of length $m$ on a graph $G$ is a sequence

$$
\left[x_{1}, e_{1}, x_{2}, e_{2}, \ldots, x_{m}, e_{m}, x_{m+1}\right] \text { with } x_{i} \in V, e_{i} \in E \text {, and } e_{i}=\left\{x_{i}, x_{i+1}\right\} \text {. }
$$

A walk is called a path if all $x_{i}$ and all $e_{i}$ are distinct. Intuitively, a saddle point on the way from $x$ to $y$ is a maximum along a walk from $x$ to $y$ that is as low as possible. In a graph-theoretical setting one usually works with paths instead of walks. However, in the present context there are three good reasons to use walks: (i) the concatenation of graph-theoretical paths is not always a path; (ii) a graph-theoretical walk is the discrete version of a topological path, which is the natural object to consider; (iii) the use of paths leads to some undesired exceptions such as the one described in the r.h.s. of Fig. 2.

Definition 4. Let $\mathbb{P}_{x y}$ be the set of all walks from $x$ to $y$. We say that $x$ and $y$ are mutually accessible at level $\eta$, in symbols ${ }^{2}$

$$
x \stackrel{\varphi \eta q}{\longrightarrow} y,
$$

if there is walk $\mathbf{p} \in \mathbb{P}_{x y}$ such that $f(z) \leq \eta$ for all $z \in \mathbf{p}$, respectively.

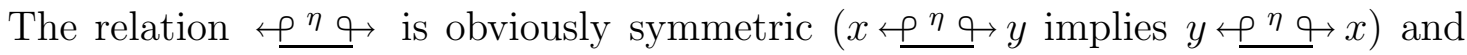

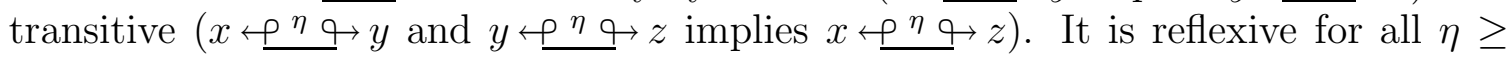
$f(x)$. The following property will be used repeatedly:

Lemma 5. For all $x, y, z \in V$ :

$$
x \stackrel{\varphi f(x) q}{\hookrightarrow} y \text { and } y \stackrel{\varphi f(y) q}{\hookrightarrow} z \text { implies } x \stackrel{\varphi f(x) q}{\hookrightarrow} z
$$

Proof. Observe that $x \stackrel{\rho f(x) q}{\hookrightarrow} y$ implies $f(y) \leq f(x)$; hence $y \underline{\rho f(x)} q z$.

Definition 6. The saddle height $\hat{f}(x, y)$ between two configurations $x, y \in V$ is the minimum height at which they are accessible from each other, i.e.,

$$
\hat{f}(x, y)=\min _{\mathbf{p} \in \mathbb{P}_{x y}} \max _{z \in \mathbf{p}} f(z)=\min \{\eta \mid x \stackrel{\varphi \eta q}{q} y\}
$$

\footnotetext{
${ }^{2}$ This symbol is dedicated to Peter Schuster, who introduced the authors not only to science but also to the art of $\mathrm{T}_{\mathrm{E}} \mathrm{X}$.
} 
In particular, we have $\hat{f}(x, x)=f(x)$. The saddle heights $\hat{f}(x, y)$ forms an ultrametric distance measure between distinct local minima, see e.g. [23, 29]. For completeness we note that the barrier of a local minimum $x \in \mathcal{M}$ is

$$
\beta(x)=\min \{+\infty ; \hat{f}(x, y)-f(x) \mid y \in \mathcal{M}, f(y)<f(x)\}
$$

Parameters such as depth and difficulty can be derived from $\beta$ and its variants [5].

2.4. Cycles. The notion of cycles, which for our purposes reduces to connected components of level sets, is central to the theory of simulated annealing, see e.g. $[5,6]$. In the literature on "disconnectivity graphs" the cycles are usually called "super-basins" [2].

Definition 7. The cycle of $x \in V$ at height $\eta, C_{\eta}(x)$, is the connected component of the level set $\{y \in V \mid f(y) \leq \eta\}$ that contains $x$.

Thus $x \in C_{\eta}(x)$ for $\eta \geq f(x)$ and $C_{\eta}(x)=\emptyset$ for $\eta<f(x)$. Obviously, we have

$$
C_{\eta}(x)=\left\{y \in V \mid y \stackrel{\varphi^{\eta} q}{\longrightarrow} x\right\}
$$

We remark that definition 7 and equ.(8) are equivalent in the case of landscapes on finite graphs. In general topological space equ.(8) refers to path-connected components while definition 7 deals with to ordinary connected components.

Lemma 8. Let $\eta^{\prime} \leq \eta^{\prime \prime}$ and $x, y \in V$. Then either $C_{\eta^{\prime}}(x) \cap C_{\eta^{\prime \prime}}(y)=\emptyset$ or $C_{\eta^{\prime}}(x) \subseteq$ $C_{\eta^{\prime \prime}}(y)$.

Proof. Suppose there is $q \in C_{\eta^{\prime}}(x) \cap C_{\eta^{\prime \prime}}(y)$. Then $\left.x \underline{\rho \eta^{\prime}} q q \underline{\varphi \eta^{\prime \prime}} q\right) y$, hence $x \stackrel{P \eta^{\prime \prime}}{\hookrightarrow} y$ and $C_{\eta^{\prime}}(x) \subseteq C_{\eta^{\prime \prime}}(x)$ because of lemma 5 .

As an immediate consequence we note:

Corollary 9. The set $\mathcal{C}(G, f)=\left\{C_{\eta}(x) \mid x \in V, \eta \in \mathbb{R}\right\}$ of the cycles of the landscape $(G, f)$ forms a hierarchy, i.e., for all $x, y \in V$ and $\eta^{\prime}, \eta^{\prime \prime} \in \mathbb{R}$ we have either $C_{\eta^{\prime}}(x) \cap$ $C_{\eta^{\prime \prime}}(y)=\emptyset, C_{\eta^{\prime}}(x) \subset C_{\eta^{\prime \prime}}(y), C_{\eta^{\prime \prime}}(y) \subset C_{\eta^{\prime}}(x)$, or $C_{\eta^{\prime}}(x)=C_{\eta^{\prime \prime}}(y)$.

2.5. Commute Points. Next we identify a set of points that is closely related to our intuition of a "saddle point".

Definition 10. The point $z \in V$ is a commute point between $x \in V$ and $y \in V$ if there is a walk $\mathbf{p} \in \mathbb{P}_{x y}$ such that

(o) $z \in \mathbf{p}$;

(i) $\max _{u \in \mathbf{p}} f(u)=\hat{f}(x, y)=f(z)$.

The set of commute points between $x$ and $y$ is denoted by $S^{*}(x, y)$.

Commute points can also be characterized in terms of the cycles introduced above.

Theorem 11. A point $s \in V$ is a commute point between $x$ and $y$ if and only if

(i) $C_{\eta}(x) \cap C_{\eta}(y)=\emptyset$ for all $\eta<f(s)$.

(ii) $C_{f(s)}(x)=C_{f(s)}(y)$

(iii) $s \in C_{f(s)}(x)$ 


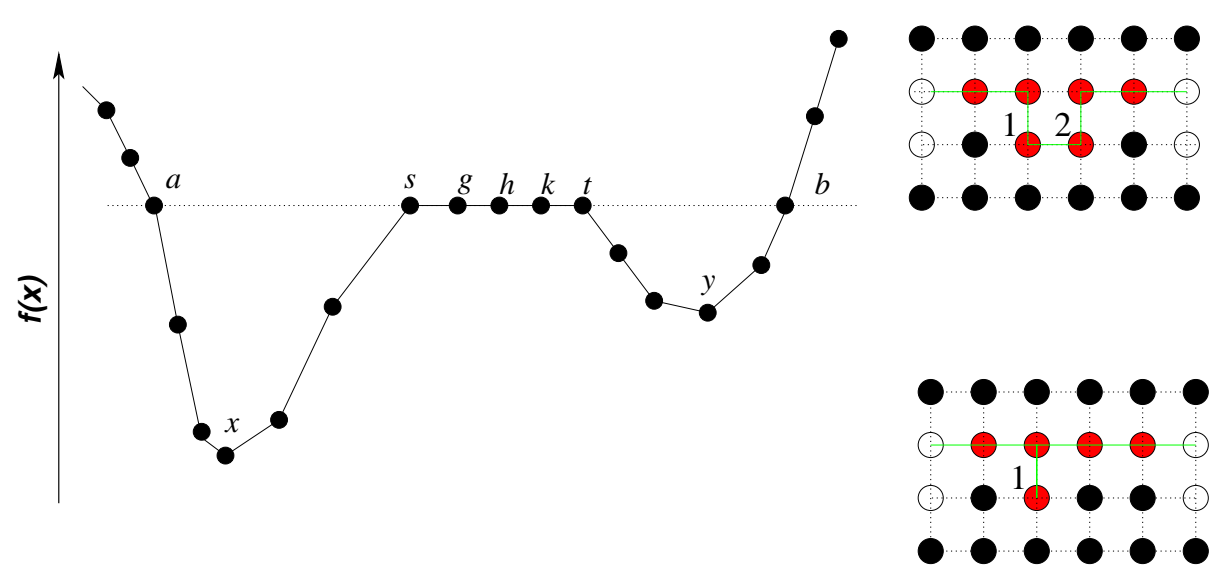

Figure 2. Saddle points in a degenerate landscape. L.h.s.: All of $a, b, g, h, k, s, t$ are commute points, $g, h, k$ are (degenerate) local minima, $g, h, k, s$ and $t$ are weak merging points (and hence also saddle points), none of these points is a (strict) merging point.

R.h.s.: $f$-values increase from white to black. The red vertices are saddle points connecting the two white basins. Replacing walks by paths in definition 15 would imply that 1 is not saddle point between $x$ and $y$ in the second situation, while it is such a saddle in the first situation. While this might be geometrically appealing it causes algorithmic difficulties since we cannot treat the flat connected part as a unit.

Proof. First suppose $s$ is a commute point between $x, y \in \mathcal{M}$. Thus $x \stackrel{p f(x)}{\longrightarrow}$. $y$ and hence $C_{f(s)}(x)=C_{f(s)}(y)$. Since $f(s)=\max _{u \in \mathbf{P}} f(u)=\hat{f}(x, y)$ we know that $x \nLeftarrow \underline{q} \not y$ for all $\eta<f(s)$, i.e., (i) holds. Condition (o) of the definition of course implies (iii) of the theorem.

Conversely, assume the conditions of the theorem. By (ii) we have $x \stackrel{\varphi^{f(s)} q}{\rightarrow} y$ and hence there is $\mathbf{p} \in \mathbb{P}_{x y}$ with height $\eta \geq f(s)$. From (i) we see that there is no such path with height $\eta<f(x)$, thus $\hat{f}(x, y)=\max _{u \in \mathbf{p}} f(u)=f(s)$. Since $s \in C_{f(s)}(x)$ we can choose $\mathbf{p}$ to run through $s$.

The definition of $S^{*}(x, y)$ might seem strange since it defines the end-points $x$ and $y$ of the walk as commute points whenever they are the highest points along some $\mathbf{p} \in \mathbb{P}_{x y}$. In particular we have $x \in S^{*}(x, x)$. These properties are, however, a significant technical convenience.

2.6. Strict Merging Points. While definition 10 above is appealing because of its (relative) simplicity, it has a major shortcoming in the degenerate case. Consider the simple 1-dimensional landscape on the l.h.s. of Figure 2. Then both $a$ and $b$ are commute points according to definition 2 , a fact that contradicts the intuitive notion of saddle points.

A different approach starts from the cycles instead of considering walks connecting local minima.

Definition 12. A point $m \in V$ is a strict merging point if there are local minima $x, y \in \mathcal{M}$ such that

(i) $C_{\eta}(x) \cap C_{\eta}(y)=\emptyset$ for all $\eta<f(m)$, 
(ii) $C_{f(m)}(x)=C_{f(m)}(y)$,

(iii) $m \in \overline{C_{\eta^{\prime}}(x)} \cap \overline{C_{\eta^{\prime \prime}}(x)}$ for some $\eta^{\prime}, \eta^{\prime \prime}<f(m)$.

We say that $m$ is a strict merging point between (the basins of) $x$ and $y$ in this case. The set of strict merging points between $x$ and $y$ will be denoted by $\hat{M}(x, y)$.

If $f$ is non-degenerate, then $m$ is a strict merging point between $x$ and $y \neq x$ if and only if it is the (uniquely determined) commute point between $x$ and $y$.

Lemma 13. If $m$ is a strict merging point then it is a commute point. If $m$ is a strict merging point, then it is not a local minimum.

Proof. From (i) and (ii) we conclude that $f(m)=\hat{f}(x, y)$. By (iii) $m$ is in particular at least a neighbor of a point $q \in C_{f(m)}(x)$ and thus itself contained in the connected component $C_{f(m)}(x)$. Hence condition (iii) of definition 11 is satisfied.

To see the second part of the lemma consider local minimum $z$. Then $C_{\eta}(z)=\emptyset$ for all $\eta<f(z)$ and hence $\overline{C_{h}(z)}=\emptyset$, and condition (iii) is never satisfied.

In a non-degenerate landscape each commute point is therefore either a local minimum or a strict merging point.

2.7. Merging Point. None of the points $s, t, g, h, k$ in the l.h.s. of Fig. 2 is a strict merging point. Thus, strict merging points are also not the desired construction. By lemma 13 we see that the desired definition must lie somewhere between commute points and strict merging points. Let us consider both avenues.

In order to weaken the definition of strict merging points we define the borderless cycle of $x$ at height $\eta$ in the following way

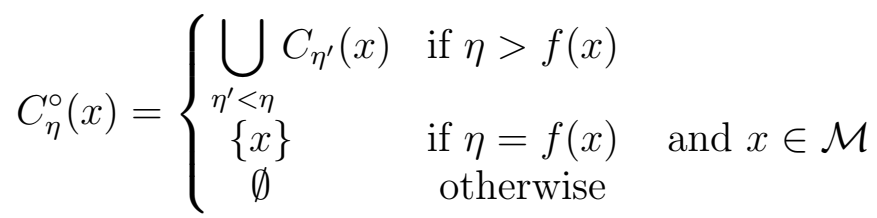

Definition 14. $m \in V$ is a merging point between the local minima $x, y \in \mathcal{M}$ if

(i) $C_{\eta}(x) \cap C_{\eta}(y)=\emptyset$ for all $\eta<f(m)$,

(ii) $C_{f(m)}(x)=C_{f(m)}(y)$

(iii) $m \in \overline{C_{f(m)}^{\circ}(x)} \cap \overline{C_{f(m)}^{\circ}(y)}$

We write $M(x, y)$ for the set of merging points between $x$ and $y$. The main difference between definitions 14 and 12 is that $z \in \mathcal{M}$ is always a merging point but never a strict merging point. To see this, choose $x=y=z$. Then (i) and (ii) is satisfied trivially, and (iii) follows from $C_{f(z)}^{\circ}(z)=\{z\}$. Furthermore, the definition now includes $s$ and $t$ in Fig. 2 as merging points between $x$ and $g$, and $k$ and $y$, respectively.

2.8. Saddle Points. The definition of a commute point includes $a$ and $b$ in Fig. 2 because nothing prevents the walk connecting $x$ with $y$ from first visiting $a$ and returning to $x$ before crossing the "true saddle" to $y$. With the help of the the following notation we can "repair" this definition. The idea is now to consider only walks that never return to a basin that they have already left. The following formalization(s) of this idea appears natural: 
Definition 15. A point $s$ is saddle point between $x$ and $y$ if there is a walk $\mathbf{p} \in \mathbb{P}_{x y}$ such that

(o) $s \in \mathbf{p}$;

(i) $\max _{u \in \mathbf{p}} f(u)=\hat{f}(x, y)=f(s)$;

(ii) For all $z \in \mathcal{M}: G\left[C_{f(s)}^{\circ}(z) \cap \mathbf{p}\right]$ is connected.

$A$ saddle point between $x$ and $y$ is direct if the walk $\mathbf{p}$ in addition satisfies

(iii) $G[\{u \in \mathbf{p} \mid f(u)=f(s)\}]$ is connected.

We write $S(x, y)$ and $\hat{S}(x, y)$ for the saddle points and direct saddle points between $x$ and $y$, respectively.

Condition (ii) ensures that $\mathbf{p}$ meets the inside of a basin not more than once. Condition (iii) means that the walk can be chosen as "unimodal", leading from $C^{\circ} f(s)(x)$ to $C^{\circ} f(s)(y)$ in such a way that $f(u)=f(s)$ for all configurations in between. The following simple result a posteriori justifies the name "saddle height" for $\hat{f}(x, y)$ introduced in definition 6 .

Lemma 16. For all $x, y \in \mathcal{M}$ there is a saddle point $s \in S(x, y)$ such that $f(s)=$ $\hat{f}(x, y)$.

Proof. The basins $C_{f(s)}^{\circ}(z)$ are connected by construction. Thus, a walk $\mathbf{p}$ satisfying (ii) can be obtained from any walk $\mathbf{p}^{\prime}$ connecting $x$ and $y$ at level $f(s)$ by replacing the part beginning at the first point $z^{\prime} \in \mathbf{p}^{\prime} \cap C_{f(s)}^{\circ}(z)$ to the last point $z^{\prime \prime} \in \mathbf{p}^{\prime} \cap C_{f(s)}^{\circ}(z)$ along $\mathbf{p}^{\prime}$ by a walk from $z^{\prime}$ to $z^{\prime \prime}$ that is contained entirely in $C_{f(s)}^{\circ}(z)$. The restriction of definition 6 to walks satisfying condition (ii) hence does not affect the saddle point height $\hat{f}(x, y)$.

Obviously, every saddle point is a direct saddle point between some basins, but not all pairs of minima are connected by a direct saddle.

Theorem 17. If $m \in V$ is a merging point then it is a saddle point. If $x \neq y$ then $M(x, y) \subseteq \hat{S}(x, y)$ for all $x, y \in \mathcal{M}$.

Proof. Let $m$ be a merging point. We have to distinguish the following cases:

(A) If $x=y$, then (i) and (ii) imply $f(m)=f(x)$ and by (iii) $m \in \overline{\{x\}}$. If $m \in \mathcal{M}$ it is also a saddle point. Otherwise there is a $u \in \mathcal{M}$ that is reached from $m$ along a gradient walk. In this case, $m$ is a strict saddle point between $x$ and $u$.

(B) If $x \neq y$ we know that $m$ is a commute point between $x$ and $y$ by theorem 11 . If $m=x$ or $m=y$ there is nothing to show since all local minima are saddle points. We even see that $m$ is a direct saddle point between $x$ and $y$. Thus, assume that $x$, $y, m$ are pairwise distinct. If $f(m)=f(x)$ then $C_{f(m)}^{\circ}(x)=\{x\}$ and by (iii) $x$ and $m$ are neighbors. The argument continues as above. Again $m$ is a direct saddle between $x$ and $y$. If $f(m)>f(x), f(y)$ then there is $p \in \partial m \cap C_{f(m)}^{\circ}(x)$ and $q \in \partial m \cap C_{f(m)}^{\circ}(x)$ and a path from $x$ to $p$ within $C_{f(m)}^{\circ}(x)$ as well as a path from $y$ to $q$ within $C_{f(m)}^{\circ}(y)$. The concatenation of these paths with the edges $(p, m)$ and $(m, q)$ is a path from $x$ to $y$ satisfying the conditions for a direct saddle. 


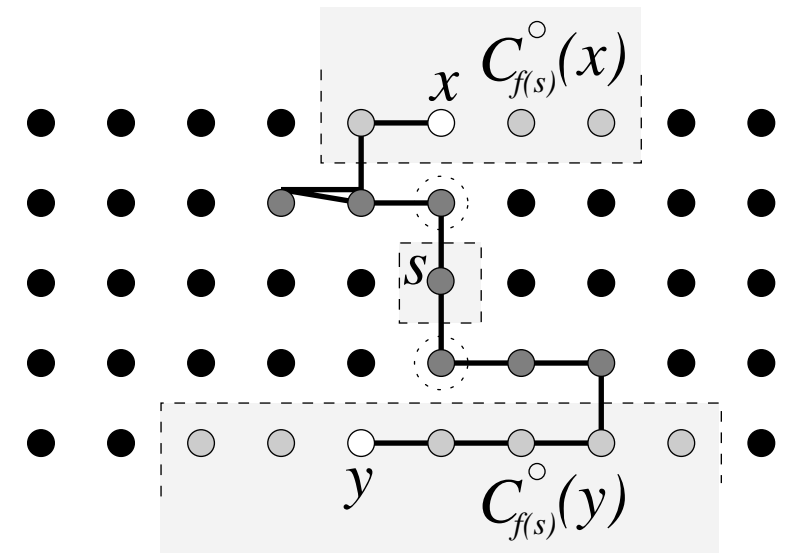

Figure 3. Not all saddle points are merging points. We indicate the values of $f$ by the darkness of the vertices on the square lattice. All dark-gray vertices are saddles (even direct saddles) between $x$ and $y$ because there is a walk from $x$ and $y$ with the necessary properties. On the other hand, only and $s$ and the two points marked with dotted circles are merging points between $x$ and $s$ or $s$ and $y$, respectively.
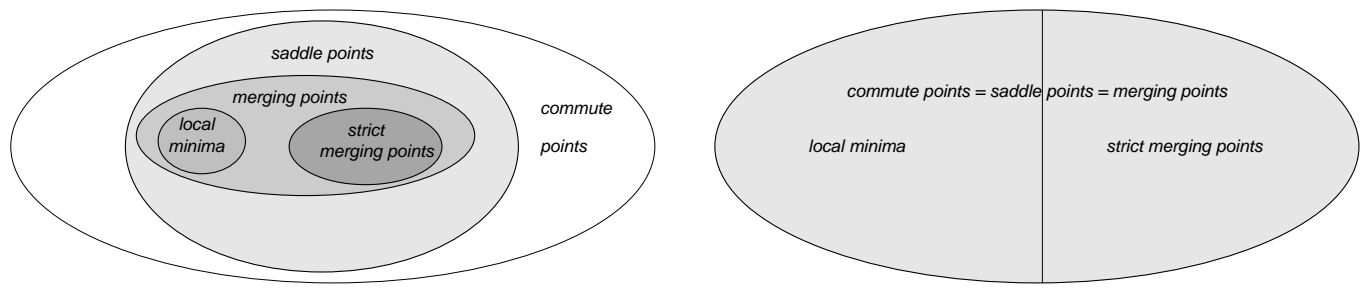

Figure 4. Venn-diagrams of the various notions of saddle points; l.h.s: degenerate landscapes, r.h.s: non-degenerate landscapes.

The converse of theorem 17 is not true in general. As an example consider the situation on a square lattice depicted in Fig 3.

2.9. Summary. In the sections above we have defined commute points, merging points, strict merging points, and saddle points in terms of conditions of the form $s$ is a $Q$-point if there are local minima $x$ and $y$ such that some conditions are satisfied. The following relationships for all $x, y \in \mathcal{M}$ follow directly from the definitions:

$$
\begin{aligned}
& \hat{M}(x, y) \subseteq M(x, y) \\
& \hat{S}(x, y) \subseteq S(x, y) \subseteq S^{*}(x, y)
\end{aligned}
$$

For $x \neq y$ we have $M(x, y) \subseteq \hat{S}(x, y)$ while $M(x, x)=\{y \in N(x) \mid f(x)=f(y)\}$ and $\hat{S}(x, x)=\{x\}$.

Naturally, we define the set of all Q-points of a landscape, where $Q$ is one of $S^{*}, S, \hat{S}, M, \hat{M}$ as

$$
Q(G, f)=\bigcup_{x, y \in \mathcal{M}} Q(x, y)
$$


The mutual relationships between these sets and the set $\mathcal{M}$ of local minima are summarized in Fig. 4.

We briefly remark that the definitions above remain meaningful for the smooth potential energy functions that are typically used in theoretical chemistry. Note, however, that in the manifold case the distinction between closure and neighborhoods becomes important.

2.10. Equivalent Saddle Points. Just as in the case of local minima it is useful to collect Q-points into equivalence classes. There appear to be two natural equivalence relations

Definition 18. Two Q-points $x, y \in Q$ are

$\leftrightarrow$ weakly equivalent if $f(x)=f(y)$ and $x \stackrel{\varphi f(x)=f(y) q}{\hookrightarrow} y$.

$\Leftrightarrow$ equivalent if they lie in the same connected component of the weak equivalence classes.

We write $Q^{*}$ and $Q(x)$ for the weak equivalence class and equivalence class of $Q$-points that contains $x$ belongs.

Note that this definition also applies to the local minima.

Lemma 19. (i) For all $x \in \mathcal{M}$ we have $\mathcal{M}(x) \subseteq S(x)$ and $\mathcal{M}^{*}(x) \subseteq S^{*}(x)$.

(ii) $\mathcal{M}(x)=\mathcal{M}^{*}(x)=S(x)=S^{*}(x)$ if and only if $\mathcal{M}(x)$ is a valley.

Proof. (i) follows immediately from the fact that every local minimum is also a saddle point.

(ii) Suppose $\mathcal{M}(x)$ is a valley, i.e., $f(y)>f(x)$ for all $y \in \partial \mathcal{M}(x)$. Consider a vertex $z \in V \backslash \mathcal{M}(x)$. Each walk $\mathbf{p}$ connecting $u \in \mathcal{M}(x)$ with $z$ must pass through a vertex $q \in \partial \mathcal{M}(x)$, hence $\hat{f}(u, z) \geq \min _{q \in \partial \mathcal{M}(x)} f(q)>f(x)$, i.e., $z \not p f(x) \not h q u$ and hence $z \notin S^{*}(x)$. Thus $S^{*}(x) \subseteq \mathcal{M}(x)$.

The converse follows from (i).

In the second part of this contribution we will show that the equivalence classes of saddle points can be computed effectively. The weak equivalence classes of saddle points, on the other hand, give rise to a hierarchical structure on $(G, f)$ which we call the barrier tree of the landscape [9].

Let us write $\mathfrak{S}=\left\{S^{*}(x) \mid x \in S\right\}$ and $\mathfrak{M}=\left\{\mathcal{M}^{*}(x) \mid x \in \mathcal{M}\right\}$ for the sets of weak equivalence classes of saddle points and local minima respectively.

\section{The Barrier Tree of a Landscape}

3.1. The Main Theorem. It is the purpose of this section to show that the set $\mathfrak{U}=\mathfrak{M} \cup \mathfrak{S}$ of equivalence classes of saddles and minima can be regarded in a very natural way as the vertex set of a tree.

We observe that the cost function $f$ is by construction constant on each set $U \in \mathfrak{U}$; hence we write $f(U)$ instead of " $f(x)$ for each $x \in U$ ". Thus the notation $U^{\prime} \stackrel{\varphi \eta q}{\eta} U^{\prime \prime}$

is also well defined. In the same vein we may write $\hat{f}\left(W, W^{\prime}\right)$ instead of " $\hat{f}\left(w, w^{\prime}\right)$ for each pair $\left(w, w^{\prime}\right) \in W \times W^{\prime}$. 
We will need the following subsets of $\mathfrak{U}$ in our discussion:

$$
\mathfrak{U}(W)=\left\{\begin{array}{lll}
\left\{W^{\prime} \in \mathfrak{U} \mid W^{\prime} \underline{\rho f(W)} \hookrightarrow, W\right\} & \text { for } & W \in \mathfrak{S} \backslash \mathfrak{M} \\
\{W\} & \text { for } & W \in \mathfrak{M}
\end{array}\right.
$$

The properties of this collections of sets is summarized in the following

Lemma 20. (i) For all $W^{\prime} \in \mathfrak{U}(W)$ we have either (a) $f\left(W^{\prime}\right)<f(W)$, or (b) $W=W^{\prime}$, or $(c) W^{\prime} \in \mathfrak{M}$ and $W^{\prime} \subset W$.

(ii) $\{\mathfrak{U}(W) \mid W \in \mathcal{U}\}$ is a hierarchy.

(iii) For all $W^{\prime}, W^{\prime \prime} \in \mathfrak{U}$ there is a unique $W \in \mathfrak{U}$ such that (1) $W^{\prime}, W^{\prime \prime} \in \mathfrak{U}(S)$ and (2) if $W^{\prime \prime \prime} \in \mathfrak{U}(W)$ and $W^{\prime}, W^{\prime \prime} \in \mathfrak{U}\left(W^{\prime \prime \prime}\right)$ then $W=W^{\prime \prime \prime}$. We have $\hat{f}\left(W^{\prime}, W^{\prime \prime}\right)=f(W)$.

Proof. (i) By construction $W^{\prime}$ is accessible from $W$ at level $f(W)$. Thus $f\left(W^{\prime}\right) \leq$ $f(W)$. If $f\left(W^{\prime}\right)<f(W)$ then $W \cap W^{\prime}=\emptyset$, i.e., none of (b) or (c) can holds. Now suppose $f(W)=f\left(W^{\prime}\right)$. If $W \in \mathfrak{M}$ then by construction $\mathfrak{U}(W)=\{W\}$, i.e., (b) holds. If $W \in \mathfrak{S} \backslash \mathfrak{M}$ then $W$ is the saddle point set of a shoulder, and hence it contains the minima set $W^{\prime}$ of the shoulder by lemma 19. Clearly $W \stackrel{\varphi f(W)}{\longrightarrow} W^{\prime}$, hence alternative (c) is satisfied. Following the argument in lemma 9 one easily verifies that $\{\mathfrak{U}(W) \mid W \in \mathcal{U}\}$ is a hierarchy. Hence for each $W^{\prime}, W^{\prime \prime} \in \mathfrak{U}$ there is an $W \in \mathfrak{U}$ such that $W^{\prime}, W^{\prime \prime} \in \mathfrak{U}(W)$ because the graph $G$ is connected. The existence and uniqueness of the minimal element $W$ is now obvious. Clearly $W$ is the set of saddle points connecting $W^{\prime}$ and $W^{\prime \prime}$, thus $f(W)$ is the height of these saddle points.

Recall that, given a collection $\mathcal{A}$ of sets, $A \in \mathcal{A}$ is a maximal subset of $B$ if $A \subseteq B$ and there is no $A^{\prime} \in \mathcal{A}$ such that $A \subset A^{\prime} \subseteq B$.

The children of $W \in \mathfrak{U}$ form the set

$$
\text { children }(W)=\left\{W^{\prime} \in \mathfrak{U}(W) \mid \mathfrak{U}\left(W^{\prime}\right) \text { is a maximal subset of } \mathfrak{U}(W) \backslash\{W\}\right\}
$$

Let $\mathfrak{T}(G, f)$ be the graph with vertex set $\mathfrak{U}$ and a directed edge $\left(W^{\prime}, W^{\prime \prime}\right) \in \mathfrak{E}$ if and only if $W^{\prime \prime} \in$ children $\left(W^{\prime}\right)$.

Theorem 21. The graph $\mathfrak{T}(G, f)$ is a rooted tree.

Proof. It follows directly from eq.(11) that $W \in \mathfrak{U}$ has a non-empty set of children if and only if $W \notin \mathfrak{M}$. Furthermore, lemma 20 implies that each $W^{\prime}$ is the child of at most one $W \in \mathfrak{U}$. Lemma 20 also implies that either $f\left(W^{\prime}\right)<f(W)$ or $W^{\prime} \in \mathfrak{M}$, in which case $W^{\prime}$ has no children. Thus $\mathfrak{T}(G, f)$ is acyclic. The hierarchy property ensures that $\mathfrak{T}(G, f)$ is a rooted forest. Finally, since $V$ is finite and $G(V, E)$ is connected we have $x \stackrel{\varphi \max f q}{\longrightarrow}$, i.e., there is $W^{*} \in \mathfrak{U}$ such that $\mathfrak{U}\left(W^{*}\right)=\mathfrak{U}$. Thus $\mathfrak{T}(G, f)$ is a connected rooted forest, i.e., a rooted tree.

We call $\mathfrak{T}(G, f)$ the barrier tree of the landscape $(G, f)$. If $f$ is non-degenerate, then each vertex of $\mathfrak{T}(G, f)$ is a set consisting of a single local minimum or saddle point. With each edge $\left(W, W^{\prime}\right)$ of $\mathfrak{T}(G, f)$ we associate the difference in the cost function $f(W)-f\left(W^{\prime}\right)$. 
3.2. Merging Graph. Closely related to the barrier tree is the merging graph of a landscape. Its vertices are the local minima and the equivalence classes $Q(x)$ of saddle points. A local minimum $y$ is connected to $Q(x)$ if there is $z \in \mathcal{M}$ and $u \in Q(x)$ such that $u$ is a merging point between $z$ and $y$. The merging graph is usually not tree. For instance, there may be more than one connected components of saddle points that merge the same two minima. Hence merging graphs have in general more nodes than the corresponding barrier trees. Of course, in a non-degenerate landscape the barrier tree and the merging graph are isomorphic.

3.3. Basins. Let $B(S)=\{x \in V \mid x \stackrel{\rho f(S) q}{\longrightarrow} S$ and $f(x)<f(S)\}$, and let $B\left(S^{\prime} ; S\right)$ be the vertex set of the connected component of $G[B(S)]$ that contains $S^{\prime}$. Thus we have $B\left(S^{\prime} ; S\right)=\emptyset$ if and only if $S^{\prime} \notin \mathfrak{U}(S) \backslash S$. The set $B\left(S^{\prime} ; S\right)$ is the basin of $S^{\prime}$ in the subtree with root $S$. We have $B\left(S^{\prime} ; S\right)=B\left(S^{\prime \prime} ; S\right)$ if and only if there is $S^{\prime \prime \prime} \in \mathfrak{U}(S) \backslash\{S\}$ such that $S^{\prime}, S^{\prime \prime} \in \mathfrak{U}\left(S^{\prime \prime \prime}\right)$, i.e., if $S^{\prime}$ and $S^{\prime \prime}$ are connected by a saddle $S^{\prime \prime \prime}$ within the basin below $S$. The basins at or below a node $S$ of the tree of course form a partition of the corresponding level sets and hence can be used to define macro-states. The problem with this approach is, however, that the partition depends explicitly on the energy level; there does not appear to be a natural way to extend such a partition of a level set to a partition of the complete state space.

A second type of basin is defined by gradient walks. In locally invertible landscapes gradient walks are uniquely defined. Hence, for each starting point $x \in V$ there is a uniquely defined end point $g(x) \in \mathcal{M}$. Naturally, we define for each $z \in \mathcal{M}$ the associated gradient basin as $G(z)=\{x \mid z=g(x)\}$. Obviously $x \in G(x)$. Furthermore, the gradient basins form a natural partition of the complete state space $V$. This partition is consistent with the barrier tree in the following sense:

$$
G(x) \cap\{y \mid f(y)<f(S)\} \subseteq B\left(S^{\prime} ; S\right) \text { for } x \in S^{\prime}
$$

That is, the part of the gradient basin of $x$ below the level $f(S)$ is contained in the basin $B\left(S^{\prime} ; S\right)$ of the minimum $x$ within the subtree with root $S$.

It appears impossible, however, to extend the notion of gradient basins unambiguously to landscapes that are not locally invertible. One possibility is to define $x \in G(z)$ if there is a instance $g$ of gradient walk (where at each step one of the degenerate directions is chosen at random) such that $z$ is the end-point of $g$. The problem with this approach is that the gradient basins do not form a partition of $V$ and hence cannot serve as macro-states.

\section{Applications}

4.1. The Program barriers. From the above results it is fairly straightforward to implement a program that constructs the barrier tree of a landscape from an energy or cost sorted list of configurations. This list may contain either all configurations or only the configurations below some threshold energy. The only part of the barriers algorithm that depends on the details of the configuration space is the routine that generates all neighbors of a configuration. Below we show a few examples of spin 
glass and RNA secondary structure landscapes. Applications to other configuration spaces are reported in [1] (phylogenetic trees) and [26] (permutations).

The barriers program first identifies local minima if it reads a conformation that has no neighbors at lower energy. Each configuration $x$ is then labeled by the lowest minimum $m$ such that $x \in C_{f(x)}(m)$; Any set of conformations with the the same energy is decomposed into its connected components. The program then checks for each component whether it is an equivalence class of saddle points. For each basin in the barrier tree the program also records the number of conformations in the basin and the partition function over them, the size and one representative conformation of each weak equivalence class of saddle points.

Because all previously read conformations have to be stored in memory, the use of barriers is limited to (partial) landscapes of modest size. On a workstation with $512 \mathrm{MB}$ of memory, for instance, landscapes with some 20 million conformations can be handled easily.

4.2. Spin Glass Landscapes. Spin glass landscapes with Gaussian interaction coefficients, such as the Sherrington-Kirkpatrick Hamiltonian

$$
\mathcal{H}(x)=\sum_{i<j} J_{i j} x_{i} x_{j}
$$

where $x_{i}= \pm 1$ is an Ising spin variable, are essentially non-degenerate (apart from the obvious symmetry $\mathcal{H}(x)=\mathcal{H}(-x)$ in the above case). However, one obtains sometimes highly degenerate landscapes when the interaction coefficients $J_{i j}$ can take only a few values, e.g. $J_{i j} \in\{-1,0,1\}$. A small example in which $J= \pm 1$ with equal probability is shown in Figure 5. Clusters of degenerate ground states in such models are discussed e.g. in [13].

The Low Autocorrelated Binary String Problem (LABSP) [3, 11] consists of finding binary strings $x$ of length $N$ over the alphabet $\{ \pm 1\}$ with low aperiodic off-peak autocorrelation $R_{k}(x)=\sum_{i=1}^{N-k} x_{i} x_{i+k}$ for all lags $k$. Typically, one uses the cost function

$$
f(x)=2 N \mathcal{H}(x)=\sum_{k=1}^{N-1}\left[\sum_{i=1}^{N-k} x_{i} x_{i+k}\right]^{2}=\frac{1}{2 N} \sum_{k=1}^{N-1} R_{k}(x)^{2} .
$$

The LABSP may serve as an example of a particularly highly degenerate landscape on the Boolean hypercube. It can be understood as the sum of a dominating 4-th order spin glass with a small quadratic contribution and interaction coefficients $J_{\ldots} \in\{0,1\}$ $[4,27]$.

A recent study [8] focused on properties of the barrier structure, such as depth and difficulty parameters $[5,12,15,24]$, which are independent of degeneracies of local minima and saddle points. Degeneracies, however, appear to play a substantial role in this landscape as the lower panel in Fig. 6 shows: a large fraction of the local minima is not strict unless $n \equiv 0 \bmod 4$. It is interesting to note, furthermore, that all ground states appear to be strict minima, at least for $n<25$. Some exact results on the ground states of the periodic version of the LABSP and their relationships to number theory are discussed in [21]. 


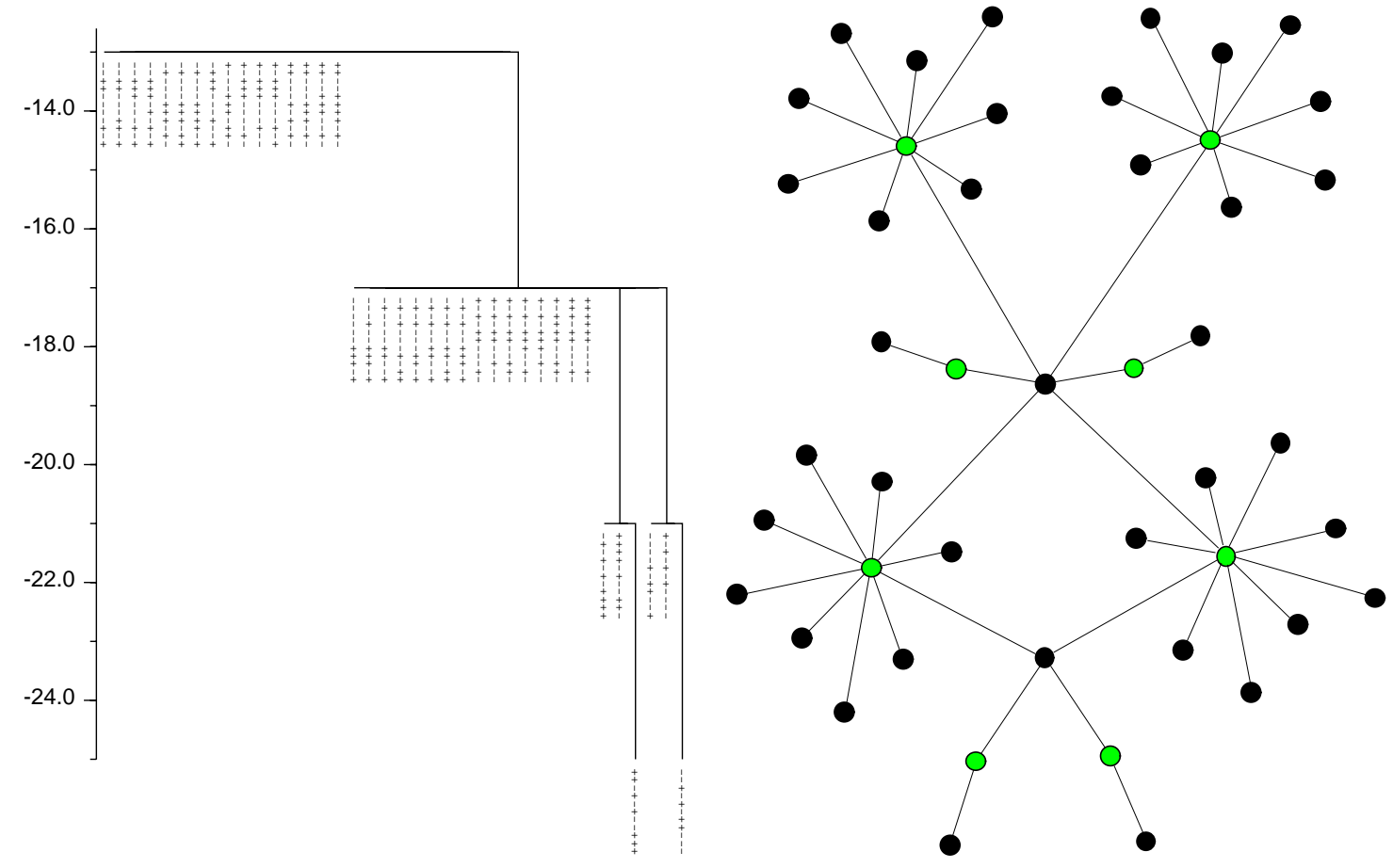

Figure 5. Barrier tree and merging graph of a quadratic spin glass, equ.(14), with $n=10$ spins and $J_{i j}= \pm 1$. In merging graphs we show local minima by in circle and saddle points in colored.

4.3. RNA Folding Landscapes. An RNA secondary structure is a list of base pairs $(i . j)$, where any base $i$ may pair with at most one other base $j$ and there are no two pairs $(i . j)$ and $(k . l)$ with $i<k<j<l$ (no pseudo-knots). The folding landscape of an RNA molecule is obtained as follows: The vertices are the secondary structures that are compatible with the molecule's sequence; the edges are defined by a move set, typically the addition or removal of a single base pair from the structure. A well-established energy model based on experimentally determined parameters [20] is used to evaluate the structures.

Optimal and near-optimal secondary structures as well as equilibrium ensembles can be predicted by means of efficient dynamic programming algorithms [14, 31, 33, 32]. Nevertheless, RNA folding landscapes are of particular interest [9], because the energy barriers for some sequences are large enough to prevent equilibration within the typical lifetime of an RNA molecule. Several experimental studies reported biological important RNA molecules with rugged folding landscapes full of kinetic traps see e.g. [28].

As an example we consider the spliced leader (SL) RNA of Leptomonas collosoma. SL RNA plays a key role in mRNA processing of trypanosomes. This small RNA molecule can potentially form two competing secondary structures (a meta-stable and the ground state conformation) of nearly equal free energy. The molecule is known to act as a conformational switch $[18,19]$. 

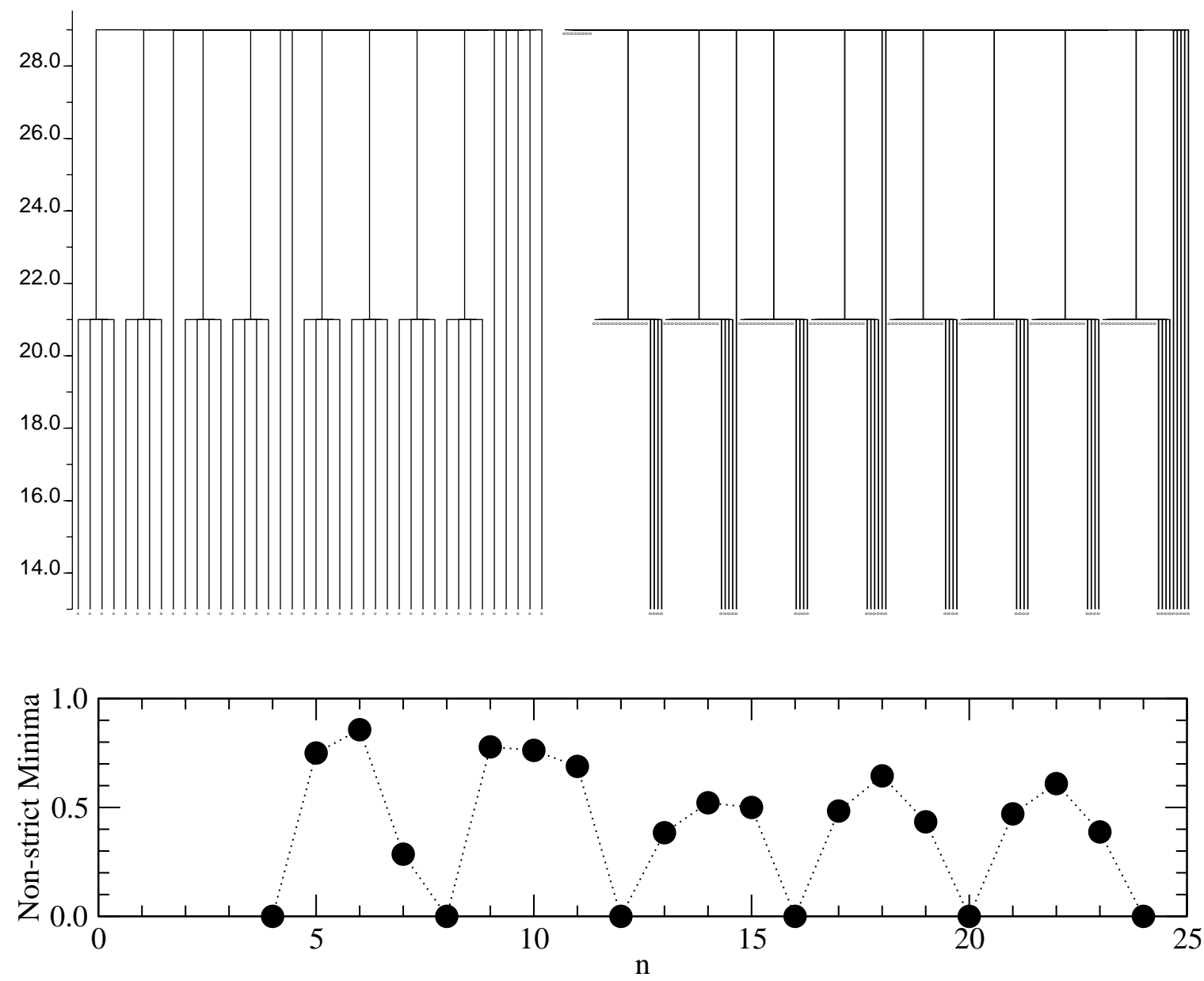

Figure 6. Barrier tree of the Low Autocorrelated Binary String Problem, $n=10$. The l.h.s. shows only the 40 strict minima (all of which are global minima) and their saddle points, while the tree on the r.h.s. contains all 168 minima. We see a number of minima that are parts of shoulders at both saddle-point levels. Local minima are indicated by little dots. The panel below shows the fraction of non-strict local minima as a function of the system size.

Fig. 7 contrasts an almost non-degenerated landscape (RNA example) to a degenerated one (LABSP example). The left part of Fig. 7 shows the barrier trees of SL RNA (upper) and LABSP (lower) and the right part the corresponding merging graphs. The barrier tree of SL RNA is restricted to the first 100 local minima. The basins of attraction of the two alternative conformations are separated by a high barrier. In the merging graph of SL RNA two major star-like components corresponding to the two "basins of attraction", the meta-stable state (upper one) and the ground state (lower one) can be recognized.

In the RNA example the merging graph and the barrier tree are very similar. The only difference are a few degenerate saddle connections where two or more connected components of saddle points connect the same two minima. In the LABSP example, 

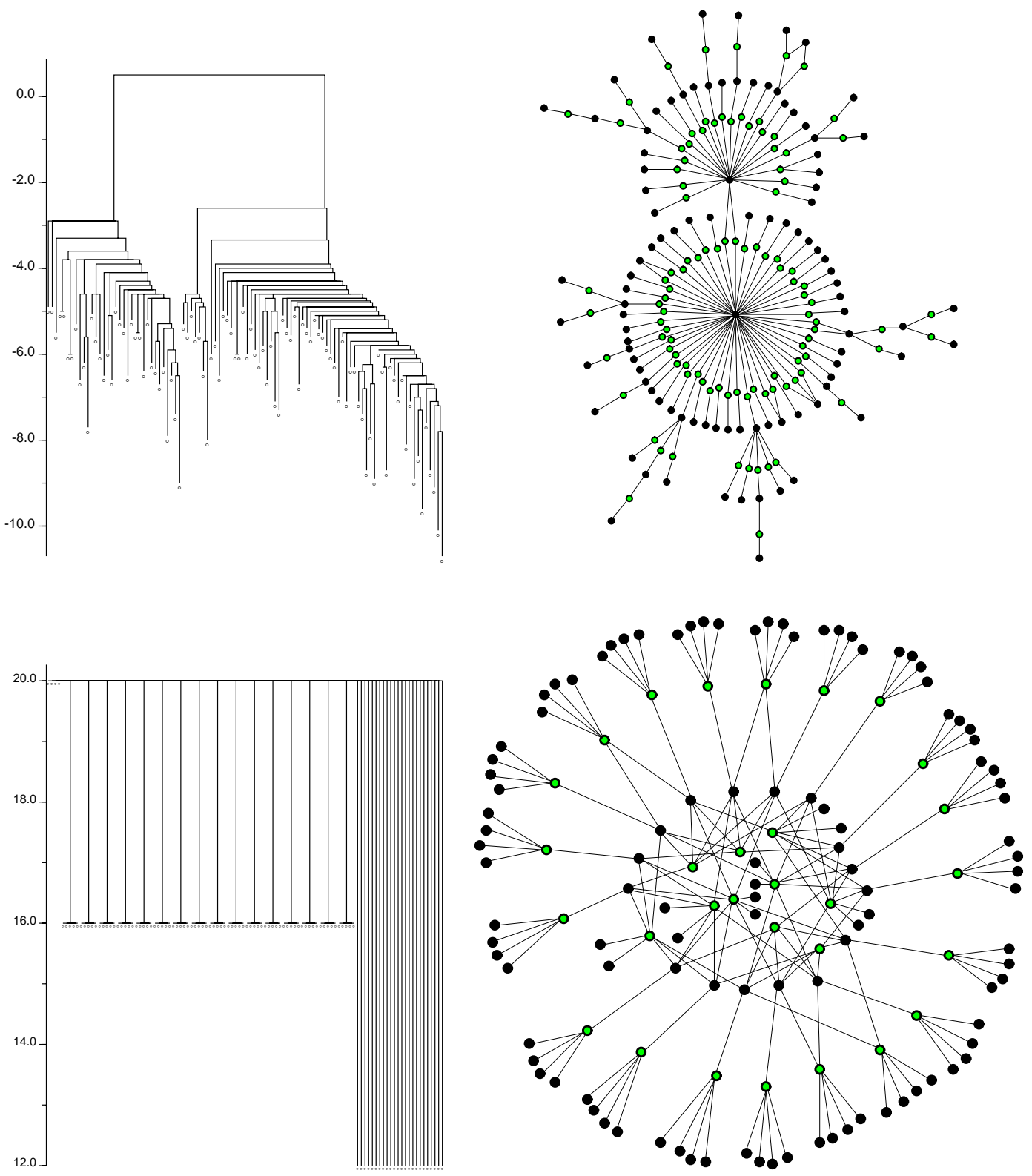

Figure 7. Energy Landscape of SL RNA of Leptomonas collosoma (upper panels) compared to the landscape of the LABSP with $n=9$ (lower panels). We show the barrier trees (l.h.s.) and the merging graphs (r.h.s.) for comparison. The RNA example is restricted to 100 lowest local minima, the LABSP example shows the complete landscape.

on the other hand, the highly degenerate interior nodes of the barrier tree are resolved into a complicated network.

\section{Discussion}

Barrier trees summarize salient features of a landscape and help interpret and understand the kinetics on the landscape without resorting to lengthy simulations. 
Furthermore, they provide a convenient starting point for a coarse grained description of the dynamics. In this work we have extended the concept of barrier trees to degenerate landscapes. Such landscapes may contain large numbers of shallow minima without a barrier which may nevertheless act as entropic traps. Similarly, unique saddle points are replaced by connected classes of equivalent saddle points which collectively act as transition state between basins. Thus, including degeneracies in the analysis is a first step toward inclusion of entropic terms in a coarse grained dynamics. The barriers program introduced here can be easily adapted to arbitrary landscapes. The example landscapes shown above exhibit very different degrees of degeneracy, and the level of degeneracy may provide a useful classification criterion.

Acknowledgments. This work was performed in part during a stay at ZIF in Bielefeld in May 2001 as part of the working group The Sciences of Complexity: From Mathematics to Technology to a Sustainable World. Useful comments by Stefan Wuchty are gratefully acknowledged.

\section{References}

[1] O. Bastert, D. Rockmore, P. F. Stadler, and G. Tinhofer. Landscapes on spaces of trees. Appl. Math. Comput., 2001. in press, SFI preprint 01-01-006.

[2] O. M. Becker and M. Karplus. The topology of multidimensional potential energy surfaces: Theory and application to peptide structure and kinetics. J. Chem. Phys., 106:1495-1517, 1997.

[3] J. Bernasconi. Low autocorrelation binary sequences: statistical mechanics and configuration space analysis. J. Physique, 48:559-567, 1987.

[4] J. P. Bouchaud and M. Mézard. Self-induced quenced disorder: A model for the glass transition. J. Physique I France, 4:1109-1114, 1994.

[5] O. Catoni. Rough large deviation estimates for simulated annealing: Application to exponential schedules. Ann. Probab., 20:1109-1146, 1992.

[6] O. Catoni. Simulated annealing algorithms and Markov chains with rate transitions. In J. Azema, M. Emery, M. Ledoux, and M. Yor, editors, Seminaire de Probabilites XXXIII, volume 709 of Lecture Notes in Mathematics, pages 69-119. Springer, Berlin/Heidelberg, 1999.

[7] J. P. Doye, M. A. Miller, and D. J. Welsh. Evolution of the potential energy surface with size for Lennard-Jones clusters. J. Chem. Phys., 111:8417-8429, 1999.

[8] F. F. Ferreira, J. F. Fontanari, and P. F. Stadler. Landscape statistics of the low autocorrelated binary string problem. J. Phys. A: Math. Gen., 33:8635-8647, 2000.

[9] C. Flamm, W. Fontana, I. Hofacker, and P. Schuster. RNA folding kinetics at elementary step resolution. RNA, 6:325-338, 2000.

[10] P. Garstecki, T. X. Hoang, and M. Cieplak. Energy landscapes, supergraphs, and "folding funnels" in spin systems. Phys. Rev. E, 60:3219-3226, 1999.

[11] M. J. E. Golay. Sieves for low-autocorrelation binary sequences. IEEE Trans. Inform. Th., IT-23:43-51, 1977.

[12] B. Hajek. Cooling schedules for optimal annealing. Math. Operations Res., 13:311-329, 1988.

[13] A. K. Hartmann. Ground-state clusters of two, three and four-dimensional $\pm j$ Ising spin glasses. Phys. Rev. E, 63:016106 (7pages), 2001.

[14] I. L. Hofacker, W. Fontana, P. F. Stadler, S. Bonhoeffer, M. Tacker, and P. Schuster. Fast folding and comparison of RNA secondary structures. Monatsh. Chemie, 125:167-188, 1994.

[15] W. Kern. On the depth of combinatorial optimization problems. Discr. Appl. Math., 43:115-129, 1993.

[16] T. Klotz and S. Kobe. Exact low-energy landscape and relaxation phenomena in Ising spin glasses. Acta Physica Slovaca, 44:347-356, 1994. 
[17] T. Klotz and S. Kobe. "Valley Structures" in the phase space of a finite 3D Ising spin glass with $\pm i$ interactions. J. Phys. A: Math. Gen, 27:L95-L100, 1994.

[18] K. A. LeCuyer and D. M. Crothers. The Leptomonas collosoma spliced leader RNA can switch between two alternate structural forms. Biochemistry, 32:5301-5311, 1993.

[19] K. A. LeCuyer and D. M. Crothers. Kinetics of an RNA conformational switch. Proc. Natl. Acad. Sci. USA, 91:3373-3377, 1994.

[20] D. Mathews, J. Sabina, M. Zucker, and H. Turner. Expanded sequence dependence of thermodynamic parameters provides robust prediction of RNA secondary structure. J. Mol. Biol., 288:911-940, 1999.

[21] S. Mertens and C. Bessenrodt. On the ground states of the Bernasconi model. J. Phys. A, 31:3731-3749, 1998.

[22] M. Mézard, G. Parisi, and M. A. Virasoro. Spin Glass Theory and Beyond. World Scientific, Singapore, 1987.

[23] R. Rammal, G. Toulouse, and M. A. Virasoro. Ultrametricity for physicists. Rev. Mod. Phys., 58:765-788, 1986.

[24] J. Ryan. The depth and width of local minima in discrete solution spaces. Discr. Appl. Math., 56:75-82, 1995.

[25] P. Schuster, W. Fontana, P. F. Stadler, and I. L. Hofacker. From sequences to shapes and back: A case study in RNA secondary structures. Proc. Royal Society London B, 255:279-284, 1994.

[26] B. M. R. Stadler and P. F. Stadler. Generalized topological spaces in evolutionary theory and combinatorial chemistry. J. Chem. Inf. Comput. Sci., 2001. in press, (Proceedings MCC 2001, Dubrovnik).

[27] P. F. Stadler. Landscapes and their correlation functions. J. Math. Chem., 20:1-45, 1996.

[28] D. Treiber and J. R. Williamson. Exposing the kinetic traps in RNA folding. Curr. Opin. Struct. Biol., 9:339-345, 1999.

[29] A. M. Vertechi and M. A. Virasoro. Energy barriers in SK spin glass models. J. Phys. France, 50:2325-2332, 1989.

[30] D. J. Wales, M. A. Miller, and T. R. Walsh. Archetypal energy landscapes. Nature, 394:758-760, 1998.

[31] S. Wuchty, W. Fontana, I. L. Hofacker, and P. Schuster. Complete suboptimal folding of RNA and the stability of secondary structures. Biopolymers, 49:145-165, 1999.

[32] M. Zuker. On finding all suboptimal foldings of an RNA molecule. Science, 244:48-52, 1989.

[33] M. Zuker and P. Stiegler. Optimal computer folding of larger RNA sequences using thermodynamics and auxiliary information. Nucleic Acids Research, 9:133-148, 1981. 